\title{
Analysis of positivity and stability of fractional discrete-time nonlinear systems
}

\author{
T. KACZOREK* \\ Faculty of Electrical Engineering, Białystok University of Technology, 45D Wiejska St., 15-351 Białystok
}

\begin{abstract}
The positivity and asymptotic stability of the fractional discrete-time nonlinear systems are addressed. Necessary and sufficient conditions for the positivity and sufficient conditions for the asymptotic stability of the fractional nonlinear systems are established. The proposed stability tests are based on an extension of the Lyapunov method to the positive fractional nonlinear systems. The effectiveness of tests is demonstrated on examples.
\end{abstract}

Key words: positive, fractional, discrete-time, asymptotic stability, nonlinear, Lyapunov method.

\section{Introduction}

A dynamical system is called positive if its trajectory starting from any nonnegative initial condition state remains forever in the positive orthant for all nonnegative inputs. An overview of state of the art in positive system theory is given in the monographs $[8,15]$ and in the papers $[16,17,19,20]$. Models having positive behavior can be found in engineering, economics, social sciences, biology and medicine, etc.

The Lyapunov, Bohl and Perron exponents and stability of time-varying discrete-time linear systems have been investigated in [1-7]. The positive standard and descriptor systems and their stability have been analyzed in [15-20]. The positive linear systems with different fractional orders have been addressed in $[17,21]$ and the descriptor discrete-time linear systems in [16]. Descriptor positive discrete-time and continuous-time nonlinear systems have been analyzed in $[10,11]$ and the positivity and linearization of nonlinear discrete-time systems by state-feedbacks in [19]. The minimum energy control of positive linear systems has been addressed in [12-14]. The stability and robust stabilization of discrete-time switched systems have been analyzed in [23, 24].

In this paper the positivity and asymptotic stability of the fractional discrete-time nonlinear systems will be investigated.

The paper is organized as follows. In section 2 the definitions and theorems concerning the positivity and stability of positive discrete-time linear systems are recalled. Necessary and sufficient conditions for the positivity of the fractional discrete-time nonlinear systems are established in section 3. The asymptotic stability of the positive fractional nonlinear systems is addressed in section 4, where the sufficient conditions for the stability are proposed. Concluding remarks are given in section 5 .

The following notation will be used: $\mathfrak{R}-$ the set of real numbers, $\mathfrak{R}^{n \times m}$ - the set of $n \times m$ real matrices, $\mathfrak{R}_{+}^{n \times m}-$ the set of $n \times m$ matrices with nonnegative entries and $\mathfrak{R}_{+}^{n}=\Re_{+}^{n \times 1}, Z_{+}$

*e-mail: kaczorek@isep.pw.edu.pl
- the set of nonnegative integers, $M_{n}$ - the set of $n \times m$ Metzler matrices (with nonnegative off-diagonal entries), $I_{n}$ - the $n \times m$ identity matrix.

\section{Positive discrete-time linear systems and their stability}

Consider the discrete-time linear system

$$
\begin{aligned}
x_{i+1} & =A x_{i}+B u_{i}, \quad i \in Z_{+}=\{0,1, . .\} \\
y_{i} & =C x_{i}+D u_{i},
\end{aligned}
$$

where $u_{i} \in \mathfrak{R}^{n}, u_{i} \in \mathfrak{R}^{m}, y_{i} \in \mathfrak{R}^{p}$ are the state, input and output vectors and $A \in \mathfrak{R}^{n \times n}, B \in \mathfrak{R}^{n \times m}, C \in \mathfrak{R}^{p \times n}, D \in \mathfrak{R}^{p \times m}$.

Definition 2.1. [8, 15] The discrete-time linear system $(2.1)$ is called (internally) positive if $x_{i} \in \mathfrak{R}_{+}^{n}, y_{i} \in \mathfrak{R}_{+}^{p}, i \in Z_{+}$for any initial conditions $x_{0} \in \mathfrak{R}_{+}^{n}$ and all inputs $x_{i} \in \mathfrak{R}_{+}^{m}, i \in Z_{+}$.

Theorem 2.1. $[\mathbf{8}, \mathbf{1 5}]$ The discrete time linear system $(2.1)$ is positive if and only if

$$
A \in \mathfrak{R}_{+}^{n \times n}, B \in \mathfrak{R}_{+}^{n \times m}, C \in \mathfrak{R}_{+}^{p \times n}, D \in \mathfrak{R}_{+}^{p \times m} .
$$

Definition 2.2. $[8,15]$ The positive discrete-time linear system (2.1) is called asymptotically stable if

$$
\lim _{i \rightarrow \infty} x_{i}=0 \text { for any } x_{0} \in \mathfrak{R}_{+}^{n}
$$

Theorem 2.2. The positive discrete-time linear system (2.1) is asymptotically stable if and only if one of the following equivalent conditions is satisfied:

1) All coefficients of the polynomial

$$
\begin{aligned}
& p_{n}(z)=\operatorname{det}\left[I_{n}(z+1)-A\right]=z^{n}+\alpha_{n-1} z^{n-1}+ \\
& +\ldots+\alpha_{1} z+\alpha_{0}
\end{aligned}
$$

are positive, i.e. $\alpha_{i}>0$ for $i=0,1, \ldots, n+1$. 
2) All principal minors of the matrix $\bar{A}=I_{n}-A=\left[\bar{\alpha}_{i j}\right]$ are positive, i.e.

$M_{1}=\left|\bar{a}_{11}\right|>0, M_{2}=\left|\begin{array}{ll}\bar{a}_{11} & \bar{a}_{12} \\ \bar{a}_{21} & \bar{a}_{22}\end{array}\right|>0, \ldots, M_{n}=\operatorname{det} \bar{A}>0$.

Proof. The proof is given in [15].

\section{Positivity of the fractional nonlinear systems}

Consider the fractional discrete-time nonlinear system

$$
\begin{aligned}
\Delta^{\alpha} x_{i} & =A x_{i}+f\left(x_{i-1}, u_{i}\right), 0<\alpha \leq 1, i \in Z_{+}=\{0,1, . .\}, \\
y_{i} & =g\left(x_{i}, u_{i}\right),
\end{aligned}
$$

where

$$
\Delta^{\alpha} x_{i}=\sum_{j=0}^{i} a_{j}^{\alpha} x_{i-j}
$$

$$
\begin{aligned}
a_{j}^{\alpha} & =(-1)^{j}\left(\begin{array}{l}
\alpha \\
j
\end{array}\right)= \\
& =(-1)^{j} \begin{cases}\frac{\alpha(\alpha-1) \ldots(\alpha-j+1)}{j !} & \text { for } j=0 \\
\frac{\alpha(\alpha)}{j=1,2, \ldots}\end{cases}
\end{aligned}
$$

is the $\alpha$-order difference of $x_{i}, x_{i} \in \mathfrak{R}^{n}, u_{i} \in \mathfrak{R}^{m}, y_{i} \in \mathfrak{R}^{p}$ are the state, input and output vectors, $A \in \mathfrak{R}^{n \times n}$ and $f\left(x_{i-1}, u_{i}\right) \in \mathfrak{R}^{n}$, $g\left(x_{i}, u_{i}\right) \in \mathfrak{R}^{p}$ are vector functions continuous in $x_{i}$ and $u_{i}$.

Note that the fractional difference (3.1c) is defined in the point "i" not as usually in the point " $i+1$ " [21, 22].

Substituting (3.1c) into (3.1a) we obtain

$$
\sum_{j=0}^{i} a_{j}^{\alpha} x_{i-j}=A x_{i}+f\left(x_{i-1}, u_{i}\right)
$$

and

$$
x_{i}=\sum_{j=1}^{i} A_{1} c_{j}^{\alpha} x_{i-j}+f_{1}\left(x_{i-1}, u_{i}\right), i \in Z_{+},
$$

where

$$
\begin{aligned}
& c_{j}^{\alpha}=-a_{j}^{\alpha}, j=1, \ldots, i, \\
& A_{1}=\left[I_{n}-A\right]^{-1} \in \mathfrak{R}^{n \times n}, f_{1}\left(x_{i-1}, u_{i}\right)=A_{1} f\left(x_{i-1}, u_{i}\right) .
\end{aligned}
$$

Assuming $x_{i}=0, i=1,2, \ldots$ from $(3.2 \mathrm{~b})$ for $i=0$ we have

$$
x_{0}=f_{1}\left(0, u_{0}\right) \text {. }
$$

Therefore, the initial condition $x_{0}$ is related with $u_{0}$ by (3.3).

Lemma 3.1. The matrix

$$
A_{1}=\left[I_{n}-A\right]^{-1} \in \mathfrak{R}_{+}^{n \times n}
$$

if and only if the positive linear system

$$
x_{i+1}=A x_{i}, A \in \mathfrak{R}_{+}^{n \times n}
$$

is asymptotically stable.
Proof. By Theorem 2.2 the positive discrete-time linear system (3.5) is asymptotically stable if and only if the matrix $A-I_{n} \in M_{n}$ is asymptotically stable (is Hurwitz) and by Theorem 2.5 the condition (3.4) is satisfied if the system (3.5) is asymptotically stable. $\square$

Theorem 3.1. The solution $x_{i}$ of the equation (3.2b) for given initial condition $x_{0} \in \mathfrak{R}^{n}$ and input $u_{i} \in \mathfrak{R}^{m}, i \in Z_{+}$has the form

$$
x_{i}=\Phi_{i} x_{0}+\sum_{j=1}^{i} \Phi_{i-j} f_{1}\left(x_{j-1}, u_{j}\right) \text {, }
$$

where

$$
\Phi_{j}=\sum_{k=1}^{j} c_{k}^{\alpha} A_{1} \Phi_{j-k}, j=1,2, \ldots, i, \Phi_{0}=I_{n} .
$$

Proof. The proof can be accomplished by induction or by checking that (3.6) satisfies the equation (3.2b). $\square$ In particular case for linear system

$$
x_{i}=\sum_{j=1}^{i} A_{1} c_{j}^{\alpha} x_{i-j}+B_{1} u_{i}, i \in Z_{+}, B_{1} \in \mathfrak{R}^{n \times m}
$$

the solution $x_{i}$ has the form

$$
x_{i}=\Phi_{i} x_{0}+\sum_{j=1}^{l} \Phi_{i-j} B_{1} u_{j}
$$

and the matrix $\Phi_{j}$ is given by (3.6b).

Remark 3.1. The solution $x_{i}$ of the equation (3.2b) can be computed using the formulae (3.6) iteratively for $i=1,2, \ldots$ and substituting $x_{j-1}$ given by (3.6a) into the vector function $f_{1}\left(x_{j-1}, u_{j}\right)$ for $i=1,2, \ldots$.

Definition 3.1. The fractional discrete-time nonlinear system (3.1) is called (internally) positive if $x_{1} \in \mathfrak{R}_{+}^{n}, y_{i} \in \mathfrak{R}_{+}^{p}, i \in Z_{+}$ for any initial conditions $x_{0} \in \mathfrak{R}_{+}^{n}$ and all inputs $u_{i} \in \mathfrak{R}_{+}^{m}, i \in Z_{+}$.

Theorem 3.2. The fractional discrete-time nonlinear system (3.1) is positive if and only if $0<\alpha \leq 1$ and the matrix $A \in \mathfrak{R}_{+}^{n \times n}$ is asymptotically stable and

$$
\begin{gathered}
f\left(x_{i-1}, u_{i}\right) \in \mathfrak{R}_{+}^{n} \text { for } x_{i} \in \mathfrak{R}_{+}^{n} \text { and } u_{i} \in \mathfrak{R}_{+}^{m}, i \in Z_{+}, \\
g\left(x_{i}, u_{i}\right) \in \mathfrak{R}_{+}^{p} \text { for } x_{i} \in \mathfrak{R}_{+}^{n} \text { and } u_{i} \in \mathfrak{R}_{+}^{m}, i \in Z_{+}
\end{gathered}
$$

Proof. Sufficiency. By Lemma 3.1 if $A \in \Re_{+}^{n \times n}$ is asymptotically stable then $A_{1} \in \mathfrak{R}_{+}^{n \times n}$. It is well-known [21] that if $0<\alpha \leq 1$ then $c_{j}^{\alpha}>0$ for $j=1,2, \ldots$. Therefore, from (3.6b) we have $\Phi_{j} \in \mathfrak{R}_{+}^{n \times n}$ for $j=0,1,2, \ldots$ and from (3.6a) $x_{i} \in \mathfrak{R}_{+}^{n}$ for $i=1,2, \ldots$ since by assumption (3.9a) $f_{1}\left(x_{i-1}, u_{i}\right)=A_{1} f\left(x_{i-1}, u_{i}\right) \in \mathfrak{R}_{+}^{n}$ for $x_{i} \in \mathfrak{R}_{+}^{n}$ and $u_{i} \in \Re_{+}^{m}, i \in Z_{+}$. If (3.9b) holds then from (3.1b) we have $y_{i} \in \mathfrak{R}_{+}^{p}$ for $i \in Z_{+}$.

Necessity. If $f\left(x_{i-1}, u_{i}\right)=0$ then $x_{i} \in \mathfrak{R}_{+}^{n}, i \in Z_{+}$only if $A_{1} \in \mathfrak{R}_{+}^{n \times n}$ and by Lemma 3.1 implies the asymptotic stability of the ma$\operatorname{trix} A \in \mathfrak{R}_{+}^{n \times n}$. Note that $x_{i} \in \mathfrak{R}_{+}^{n}$ for $i \in Z_{+}$implies the condition (3.9a). Similarly, $y_{i} \in \mathfrak{R}_{+}^{p}$ for $i \in Z_{+}$implies the condition (3.9b). 


\section{Stability of the positive fractional nonlinear systems}

Consider the fractional discrete-time nonlinear system for zero inputs $\left(u_{i}=0\right.$ and $f\left(x_{i-1}, 0\right)=\bar{f}_{2}\left(x_{i-1}\right)$ in the form

$$
\Delta^{\alpha} x_{i}=A x_{i}+\bar{f}_{2}\left(x_{i-1}\right), \quad i \in Z_{+}, 0<\alpha \leq 1
$$

or

$$
x_{i}=\sum_{j=1}^{i} A_{1} c_{j}^{\alpha} x_{i-j}+f_{2}\left(x_{i-1}\right), i \in Z_{+}, 0<\alpha \leq 1,
$$

where

$$
f_{2}\left(x_{i-1}\right)=A_{1} \bar{f}_{2}\left(x_{i-1}\right), \quad i \in Z_{+}
$$

and $A_{1}$ is defined by $(3.2 \mathrm{c})$.

Definition 4.1. The positive fractional discrete-time nonlinear system (4.1) is called asymptotically stable in the region $D \in \mathfrak{R}_{+}^{n}$ if $u_{i} \in \mathfrak{R}_{+}^{n}, i \in Z_{+}$and

$$
\lim _{i \rightarrow \infty} x_{i}=0 \text { for } x_{0} \in D \in \mathfrak{R}_{+}^{n} .
$$

To test the asymptotic stability of the system the Lyapunov method will be used. As a candidate of the Lyapunov function we choose

$$
V\left(x_{i}\right)=c^{T} x_{i}>0 \text { for } x_{i} \in \mathfrak{R}_{+}^{n}, i \in Z_{+} \text {, }
$$

where $c \in \mathfrak{R}_{+}^{n}$ is a vector with strictly positive components $c_{i}>0$ for $i=1, \ldots, n$.

Using (4.4) and (4.2) we obtain

$$
\begin{aligned}
& \Delta V\left(x_{i}\right)= V\left(x_{i+1}\right)-V\left(x_{i}\right)=c^{T} x_{i+1}-c^{T} x_{i} \\
&=c^{T}\left[\sum_{j=1}^{i+1} A_{1} c_{j}^{\alpha} x_{i-j+1}+f_{2}\left(x_{i}\right)-\right. \\
&\left.\quad-\left(\sum_{j=1}^{i} A_{1} c_{j}^{\alpha} x_{i-j}+f_{2}\left(x_{i-1}\right)\right)\right] \\
&=c^{T}\left[\sum_{j=1}^{i} A_{1} c_{j}^{\alpha}\left(x_{i-j+1}-x_{i-j}\right)+A_{1} c_{i+1}^{\alpha} x_{0}+\right. \\
&\left.\quad+f_{2}\left(x_{i}\right)-f_{2}\left(x_{i-1}\right)\right]<0
\end{aligned}
$$

and

$$
\begin{gathered}
\sum_{j=1}^{i} A_{1} c_{j}^{\alpha}\left(x_{i-j+1}-x_{i-j}\right)+A_{1} c_{i+1}^{\alpha} x_{0}+f_{2}\left(x_{i}\right)-f_{2}\left(x_{i-1}\right)<0, \\
x_{i} \in D \in \mathfrak{R}_{+}^{n}, i \in Z_{+}
\end{gathered}
$$

since $c \in \mathfrak{R}_{+}^{n}$ is strictly positive.

Therefore, the following theorem has been proved.

Theorem 4.1. The positive fractional discrete-time nonlinear system (4.1) is asymptotically stable in the region $D \in \Re_{+}^{n}$ if the condition (4.5) is satisfied.
Example 4.1. Consider the fractional discrete-time nonlinear system (4.1) with

$$
x_{i}=\left[\begin{array}{l}
x_{1, i} \\
x_{2, i}
\end{array}\right], A=\left[\begin{array}{ll}
0.3 & 0.1 \\
0.2 & 0.4
\end{array}\right], f_{2}\left(x_{i}\right)=\left[\begin{array}{c}
x_{1, i} x_{2, i} \\
x_{2, i}^{2}
\end{array}\right] .
$$

In this case

$$
\begin{aligned}
A_{1} & =\left[I_{2}-A\right]^{-1}=\left[\begin{array}{cc}
0.7 & -0.1 \\
-0.2 & 0.6
\end{array}\right]^{-1}= \\
& =\frac{1}{0.4}\left[\begin{array}{ll}
0.6 & 0.1 \\
0.2 & 0.7
\end{array}\right]=\frac{1}{4}\left[\begin{array}{ll}
6 & 1 \\
2 & 7
\end{array}\right] \in \mathfrak{R}_{+}^{2 \times 2} .
\end{aligned}
$$

The nonlinear system is positive since the matrix $A \in \mathfrak{R}_{+}^{2 \times 2}$ is asymptotically stable and $f_{2}\left(x_{i}\right) \in \mathfrak{R}_{+}^{2}$ for all $x_{i} \in \mathfrak{R}_{+}^{2}, i \in Z_{+}^{+}$. The region $D \in \mathfrak{R}_{+}^{2}$ is defined by

$$
\begin{aligned}
& D:=\left\{x_{1, i}, x_{2, i}\right\}=\sum_{j=1}^{i} A_{1} c_{j}^{\alpha} x_{i-j+1}+A_{1} c_{i+1}^{\alpha} x_{0}-x_{i}+f_{2}\left(x_{i}\right) \\
& {\left[1.5\left(\sum_{j=1}^{i} c_{j}^{\alpha} x_{1, i-j+1}+c_{i+1}^{\alpha} x_{10}\right)+\right.} \\
& +0.25\left(\sum_{j=1}^{i} c_{j}^{\alpha} x_{2, i-j+1}+c_{i+1}^{\alpha} x_{20}\right)-x_{1, i}+x_{1, i} x_{2, i} \\
& 0.5\left(\sum_{j=1}^{i} c_{j}^{\alpha} x_{1, i-j+1}+c_{i+1}^{\alpha} x_{10}\right)+ \\
& +1.75\left(\sum_{j=1}^{i} c_{j}^{\alpha} x_{2, i-j+1}+c_{i+1}^{\alpha} x_{20}\right)-x_{2, i}+x_{2, i}^{2}
\end{aligned}
$$

Let us assume

$$
x_{10}=0.1, x_{20}=0.2, \alpha=0.5, i=4 \text {. }
$$

The region defined by (4.7) with (4.8) is shown in Fig. 1.

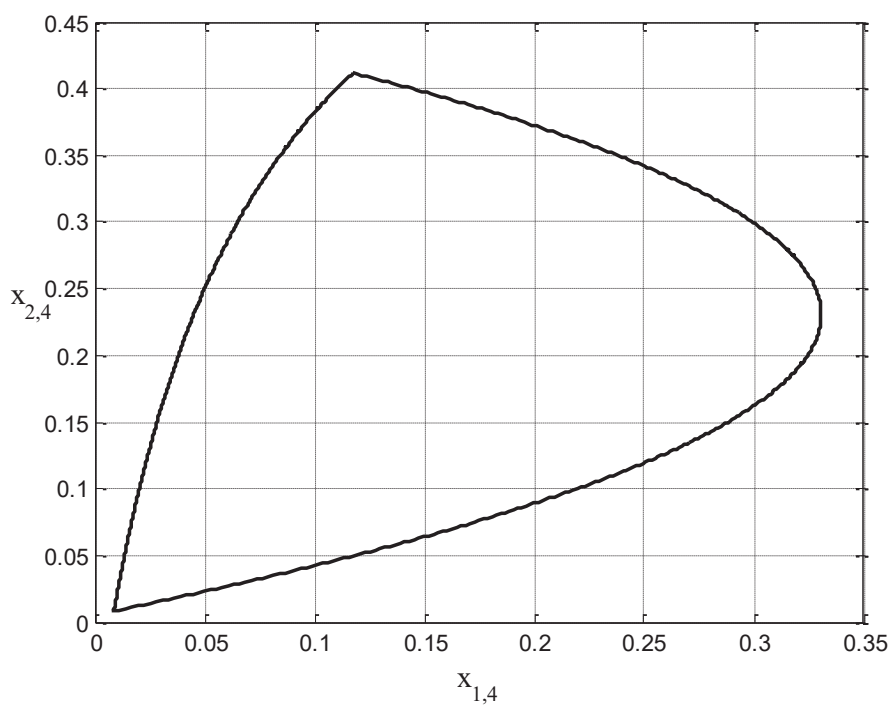

Fig. 1. Stability region (inside the curved line) 


\section{Concluding remarks}

The positivity and asymptotic stability of the fractional discrete-time nonlinear systems have been addressed. Necessary and sufficient conditions for the positivity of the fractional discrete-time nonlinear systems have been established (Theorem 3.2). Using the Lyapunov direct method, sufficient conditions for asymptotic stability of the fractional discrete-time nonlinear systems have been proposed (Theorem 4.1). The effectiveness of the conditions has been demonstrated in Example 4.1. The considerations can be extended to fractional continuous-time nonlinear systems. An open problem is an extension of the conditions to the descriptor fractional discrete-time and continuous-time nonlinear systems.

Acknowledgments. This work was supported by National Science Centre in Poland under work No. 2014/13/B/ST7/03467.

\section{REFERENCES}

[1] A. Czornik, Perturbation Theory for Lyapunov Exponents of Discrete Linear Systems, AGH University of Science and Technology Press, Kraków, 2012.

[2] A. Czornik, A. Nawrat, M. Niezabitowski, A. Szyda, "On the Lyapunov and Bohl exponent of time-varying discrete linear systems", $20^{\text {th }}$ Mediterranean Conf. on Control and Automation (MED), Barcelona, 194-197 (2012).

[3] A. Czornik, M. Niezabitowski, "Lyapunov Exponents for Systems with Unbounded Coefficients", Dynamical Systems, 28 (2), 140-153 (2013).

[4] A. Czornik, A. Nawrat, M. Niezabitowski, "On the Lyapunov exponents of a class of the second order discrete-time linear systems with bounded perturbations", Dynamical Systems, 28 (4), 473-483 (2013).

[5] A. Czornik, M. Niezabitowski, "On the stability of discrete time-varying linear systems", Nonlinear Analysis. Hybrid Systems, 9, 27-41 (2013).

[6] A. Czornik, M. Niezabitowski, "On the stability of Lyapunov exponents of discrete linear system", Proc. of European Control Conf., Zurich, 2210-2213 (2013).

[7] A. Czornik, J. Klamka, M. Niezabitowski, "On the set of Perron exponents of discrete linear systems", Proc. of World Congress of the $19^{\text {th }}$ International Federation of Automatic Control, 11740-11742 (2014).

[8] L. Farina, S. Rinaldi, Positive Linear Systems; Theory and Applications, J. Wiley, New York, 2000.
[9] T. Kaczorek, "Analysis of positivity and stability of discrete-time and continuous-time nonlinear systems", Computational Problems of Electrical Engineering, 5 (1) (2015).

[10] T. Kaczorek, "Descriptor positive discrete-time and continuous-time nonlinear systems", Proc. of SPIE, 9290, doi:10.1117/12.2074558 (2014).

[11] T. Kaczorek, "Descriptor standard and positive discrete-time nonlinear systems", Automatyzacja Procesów Dyskretnych, 1, $113-120$ (2014).

[12] T. Kaczorek, "Minimum energy control of descriptor positive discrete-time linear systems", COMPEL, 33 (3), 976-988 (2014).

[13] T. Kaczorek, "Minimum energy control of fractional descriptor positive discrete-time linear systems", Int. J. Appl. Math. Sci., 24 (4), 735-743 (2014).

[14] T. Kaczorek, "Necessary and sufficient conditions for minimum energy control of positive discrete-time linear systems with bounded inputs", Bull. Pol. Ac.: Tech., vol. 62 (1), 85-89 (2014).

[15] T. Kaczorek, Positive $1 D$ and 2D systems, Springer Verlag, London, 2001.

[16] T. Kaczorek, "Positive descriptor discrete-time linear systems", Problems of Nonlinear Analysis in Engineering Systems, vol. 1, no. 7, 1998, 38-54.

[17] T. Kaczorek, "Positive linear systems consisting of n subsystems with different fractional orders", IEEE Trans. Circuits and Systems, vol. 58, no. 6, 2011, 1203-1210.

[18] T. Kaczorek, "Positive singular discrete time linear systems", Bull. Pol. Ac.: Tech., 45(4), 619-631 (1997).

[19] T. Kaczorek, "Positivity and linearization of a class of nonlinear discrete-time systems by state feedbacks", Logistyka, 6, 5078-5083 (2014).

[20] T. Kaczorek, "Positivity and stability of discrete-time nonlinear systems", IEEE 2nd International Conference on Cybernetics, 156-159 (2015).

[21] T. Kaczorek, Selected Problems of Fractional Systems Theory, Springer-Verlag, Berlin, 2012.

[22] P. Ostalczyk, "Discrete Fractional Calculus. Selected Applications in Control and Image Processing", Series in Computer Vision, 4 (2016).

[23] H. Zhang, D. Xie, H. Zhang, G. Wang, "Stability analysis for discrete-time switched systems with unstable subsystems by a mode-dependent average dwell time approach", ISA Transactions, 53, 1081-1086 (2014).

[24] J. Zhang, Z. Han, H. Wu, J. Hung, "Robust stabilization of discrete-time positive switched systems with uncertainties and average dwell time switching", Circuits Syst. Signal Process., 33, 71-95 (2014). 